\title{
Tres marcadores calendáricos en Cocotitlán, Edo. de México
}

\author{
Stanisław Iwaniszewski
}

\begin{abstract}
o
En general las cruces punteadas o los "marcadores teotihuacanos" consisten de un par de círculos concéntricos (a veces reemplazado por los cuadrados concéntricos) centrado en un par de ejes ortogonales, plasmados en pisos estucados o en afloramientos rocosos durante el periodo Clásico en Mesoamérica. En el artículo se estudian tres marcadores recientemente descubiertos en la región de Cocotitlán, en la parte sur de la Cuenca México. El análisis revela el significado calendárico-astronómico potencial de los arreglos de agujeros y ejes que conforman esas figuras.
\end{abstract}

Palabras clave: marcadores teotihuacanos, Cocotitlán, alineamientos astronómicos, año comuptacional de 364 días

\begin{abstract}
THREE CALENDRICAL MARKERS FROM COCOTITLÁN, STATE OF MEXICO

Pecked-cross figures ("Teotihuacan markers") usually consist of a pair of concentric rings (sometimes replaced by double squares) centered on a pair of orthogonal axes, pecked into the plaster floors of the buildings or carved in rock outcrops in the Classic Period Mesoamerica. The paper examines three newly discovered cross-circle figures in the region of Cocotitlan located in the southern part of the Basin of Mexico. The study reveals the potential calendric-astronomical significance of the cups and axes producing those figures.
\end{abstract}

Keywords: Teotihuacan markers, Cocotitlán, astronomical alignments, computational year of 364 days

Stanisław Iwaniszewski - Posgrado en Arqueología, Escuela Nacional de Antropología e Historia Instituto Nacional de Antropología e Historia, México. correo: siwanisz@yahoo.com 


\section{Introducción}

Las cruces punteadas (pecked cross) son los diseños plasmados en pisos estucados o afloramientos rocosos compuestos de uno a tres círculos concéntricos centrados a una cruz (o al eje cartesiano). Su diseño está hecho a base de cavidades punteadas. Estos motivos han sido interpretados como símbolos de la división cuatripartita del espacio, instrumentos astronómico-calendáricos, marcadores topográficos o tableros para jugar. En la literatura arqueoastronómica reciben comúnmente el nombre de "marcadores astronómicos", "cruces punteadas", o "cruces-círculos".

Varios de los marcadores astronómicos encontrados en Teotihuacán, Xihuingo y Uaxactún en los estratos arqueológicamente fechables, fueron atribuidos al periodo Clásico (200 - 750 d.C., véanse von Winning 1987: 61; Cabrera Castro 2002: 202). Cabe recalcar que de acuerdo con los criterios metodológicos adoptados en este trabajo (Iwaniszewski 1991, 2005) podemos sugerir que los conjuntos de las cavidades colocadas en los círculos representarían intervalos calendáricos y que la colocación de las cruces punteadas en diferentes lugares en la Cuenca de México debe reflejar la intención de registrar ciertos ciclos o intervalos calendáricos adoptados a esa localidad. La distribución de los motivos en afloramientos rocosos situados cerca de los terrenos aptos para el cultivo de maíz puede sugerir que los agujeros representaron los intervalos calendáricos de una u otra forma asociados a los ritmos del medio ambiente natural y/o al ciclo agrícola. Es importante señalar que los agujeros fueron colocados en dos patrones distintos: unos conformaron los círculos y otros - los ejes. La tarea de evaluar su potencial calendárico-astronómico es entonces de doble naturaleza. Puesto que los marcadores por lo general constan de los elementos lineales (ejes) y circulares (círculos), suponemos que los agujeros situados en los círculos representan cuentas calendáricas y los agujeros acomodadas en los ejes indican la idea de la direccionalidad ${ }^{1}$. Los orificios colocados intersecciones de brazos y círculos se asignan como pertenecientes a los ejes. A su vez, los ejes parecen privilegiar ciertas direcciones convirtiendo todas las figuras en marcadores del paisaje o en los alineamientos celestes. Es posible que estas figuras se vinculan con las actividades desarrolladas por la élite intelectual (sacerdotes-especialistas en calendario, sacerdotesastrónomos, $\mathrm{o}$ adivinos).

El presente trabajo trata de los marcadores localizados en el municipio de Cocotitlán, en el sur de la Cuenca de México. Se concentra en el estudio de tres figuras denominadas como COC 1 , COC 2 y COC 3 . El objetivo principal es evidenciar, mediante el estudio de los marcadores, un patrón de las prácticas rituales basadas en el conteo astronómico-calendárico. 


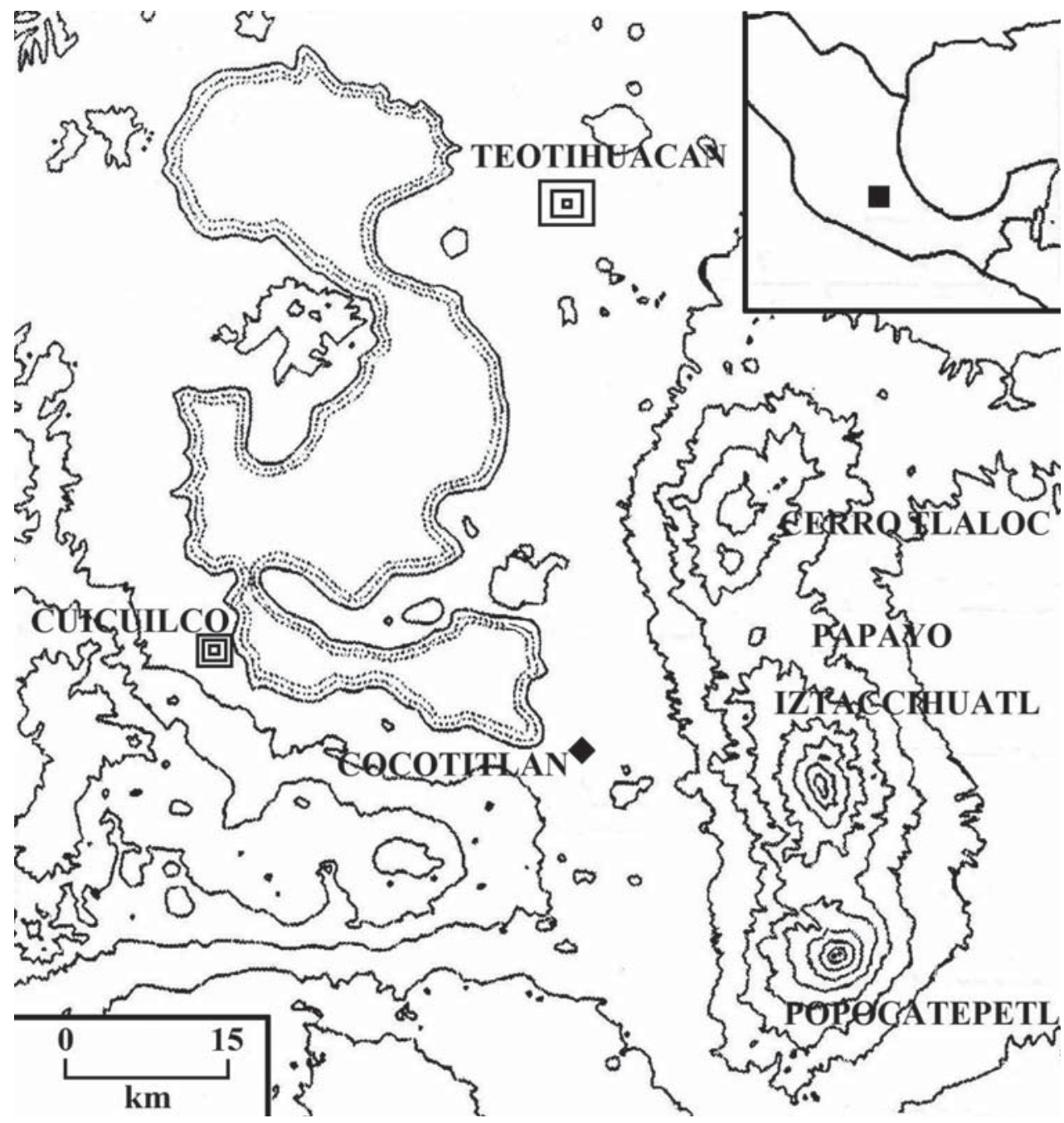

Figura 1. Localización de Cocotitlán en la Cuenca de México.

\section{Cocotitlán}

El Cerro Cocotitlán (2420 msnm), en donde en la actualidad se asienta la cabecera municipal que lleva el mismo nombre, se sitúa en la porción suroriental de la Cuenca de México, entre Chalco al oeste y Tlalmanalco al este, en el área conocida tradicionalmente como Chalco-Amecameca (véase Fig. 1). El cerro se encuentra entre los lechos de dos ríos descendientes de la Sierra Nevada: Tlalmanalco (La Compañía) al oriente y Amecameca (La Asunción) al poniente y está rodeado por las tierras idóneas para la agricultura del temporal (Galicia Cordova 1997: 20). El tipo del clima es templado subhúmedo $\mathrm{C}(\mathrm{w} 1)(\mathrm{w})$. La temporada de lluvias comienza a fines de mayo 


\section{Tlalmanalco, datos climatológicos y agrícolas}

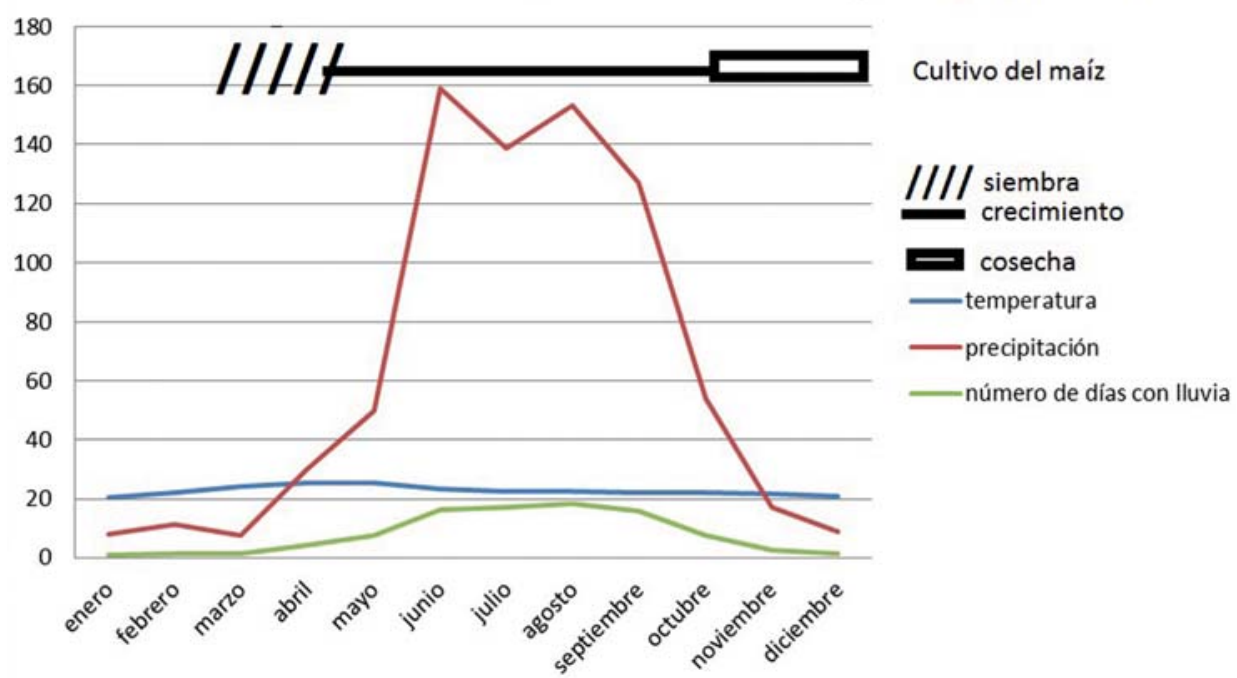

Figura 2. Tlalmanalco, datos climáticos y agrícolas.

y termina en octubre, en mayo terminan las últimas heladas y reaparecen a finales de octubre (Galicia Cordova 1997: 24; Vivó et. al. 1946: 34-35; también Fig. 2). En verano se observa el fenómeno de la sequía interestival ("canícula") que se caracteriza por la disminución de las cantidades de lluvia en julio y agosto lo que es típico para el clima en el Altiplano (véase Fig. 2).

El calendario agrícola tradicional marca dos temporadas de intensas actividades. Las siembras inician a finales de marzo y se continúan durante la primera mitad de abril, más o menos a un mes y medio antes del inicio de la temporada de lluvias (consúltese Fig. 2). Las cosechas comienzan al inicio de noviembre (después del Día de los Muertos) y se prolongan hasta fines de diciembre. Terminada la cosecha, se dan barbechos y pasos de rastra o vigadas con el fin de eliminar el crecimiento de malas hierbas y para absorber la humedad proveniente de las lloviznas y heladas (Díaz Cisneros y Cruz López 1993). Las mazorcas se desecan, deshojan y desgranan en casas durante los meses de invierno. En suma, las actividades en el campo inician con la elección de la parcela y la bendición de las semillas, luego siguen la siembra, la petición de lluvias, las labores (la primera labor (deshierbo) a 30 días, la segunda a 4550 días, la tercera ("cajoneada") a 90-100 días después de la siembra) y los primeros elotes para terminar con la cosecha, el barbecho y vigadas.

\section{COC 1}

El marcador denominado como COC 1 se localiza sobre un promontorio rocoso situado al sur del Cerro Cocotitlán, en el lugar conocido como el Calvario o la 
Capilla. Galicia Cordova (1997: 68, 104-105) reporta que los habitantes de Cocotitlán celebraban en este lugar la escenificación de la crucifixión de Cristo en el Viernes Santo. El Calvario es un promontorio aplanado rodeado por grandes afloramientos de roca. El lugar central de El Calvario lo ocupa una capilla sencilla, posiblemente erigida durante la segunda mitad del siglo XVIII (Galicia Cordova 1997: 105). En los enormes bloques de roca que bordean la superficie plana con la capilla mencionada, se encuentran varias manifestaciones rupestres lo que sugiere que fueron plasmados para demarcar los espacios necesarios para la ejecución y exhibición de las actividades rituales específicas. Los petroglifos de El Calvario, Cocotitlán fueron estudiados por Tello Charles (1993) y descritos brevemente por Meléndez García (2008). Se identificaron las imágenes de la cara de Tláloc, representaciones de yacameztli (nariguera en forma de luna), numerosas pocitas, diseños fitomorfos, etc. Algunos de esos diseños estilísticamente se clasifican como mexicas (aztecas). Es posible que formaran parte del recinto ceremonial mexica denominado Cócotl en donde se celebraban las ceremonias de sacrificios de niños durante el mes Atlcahualo (Sahagún 1979: 99 (Lib. II, Cap. XXX.12); Aveni 1991: 68-69; Broda 1991: 93-94). Aunque en la actualidad el marcador COC-1 coexiste con un complejo iconográfico más amplio, es probable que represente otra temporalidad. La evidencia arqueológica indica una continuidad ritual asociada al uso del cerro, sugiriendo que persistió el interés en utilizar este lugar para ciertos ritos propiciatorios.

En la falda sureste de El Calvario, a $2341 \mathrm{msnm}$, menos de 10 metros debajo de la cota altitudinal del área nivelada, en donde se levanta la capilla, se encontró y documentó el afloramiento rocoso con el marcador COC-1. Por primera vez este marcador fue reportado por Meléndez García (2008: 98). Se trata de una figura que consta de tres círculos concéntricos, centrados a una figura creada por 8 brazos (véase Fig. 3a). En cuanto a las dimensiones de los elementos, los orificios que constituyen dos círculos interiores y los ejes son en promedio 2 veces mayores $(2-2.5 \mathrm{~cm})$ que los del círculo exterior $(1-1.3 \mathrm{~cm})$. Los ejes miden aproximadamente entre 77 y $86 \mathrm{~cm}$, el diámetro del círculo exterior mide entre 84 y $72 \mathrm{~cm}$, del círculo intermedio alcanza entre 61 y $63 \mathrm{~cm}$ y del interior tiene $49 \mathrm{~cm}$. En suma, el área del diseño tiene un tamaño muy reducido y menor del espacio ocupado por los marcadores teotihuacanos (Aveni 1989: 94). Ya que los orificios del círculo exterior son en promedio 2 veces menores que los de la parte inferior de la figura, es posible que fueron ejecutados posteriormente.

El marcador se encuentra en la parte inferior de la roca, cerca del nivel del suelo en una superficie aplanada, tallada e inclinada (sobre el eje NO-SE la inclinación es entre $10^{\circ}$ y $12^{\circ}$ ). El acceso al marcador es fácil ya que solo se debe descender partiendo del espacio aplanado de El Calvario. La colocación del marcador a nivel del suelo implica que la mejor posición para mirarlo de frente consiste en pararse del lado sureste. Sin embargo, mirando de frente implica colocarse dando la espalda a la vista panorámica que se extiende del lugar. Adicionalmente se observa que las dimensio- 


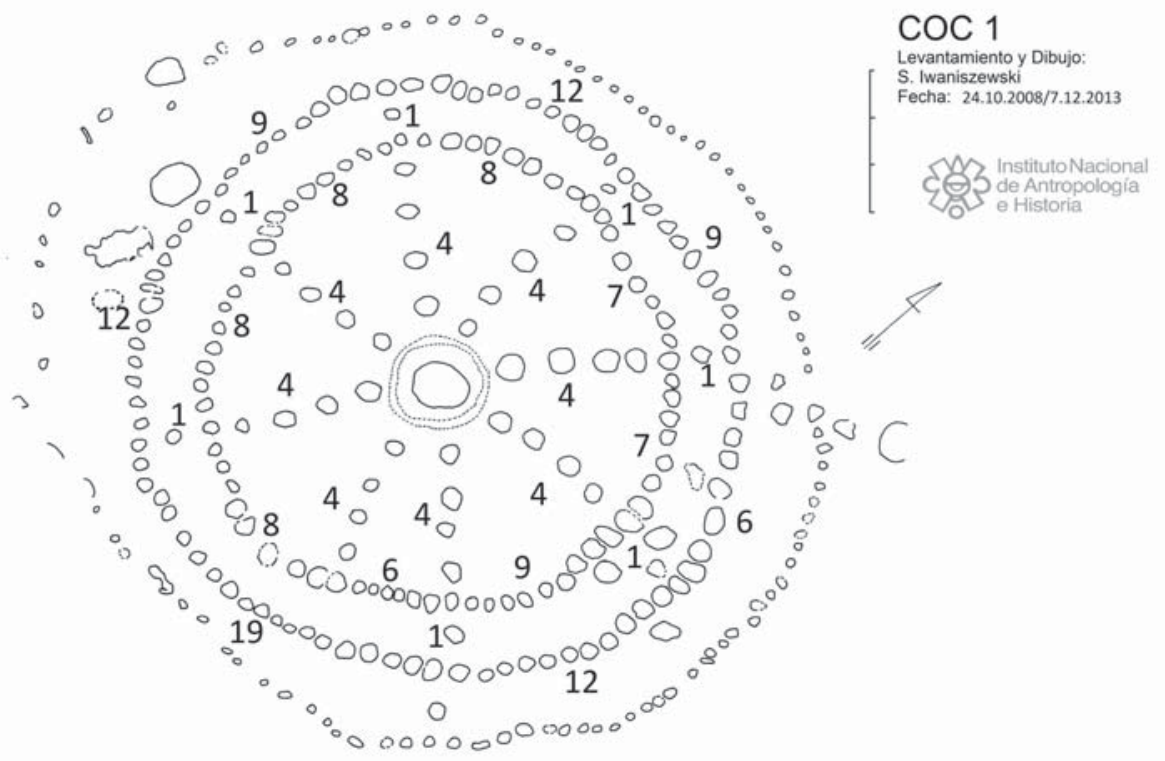

Figura 3a. El Diseño del marcador COC1.

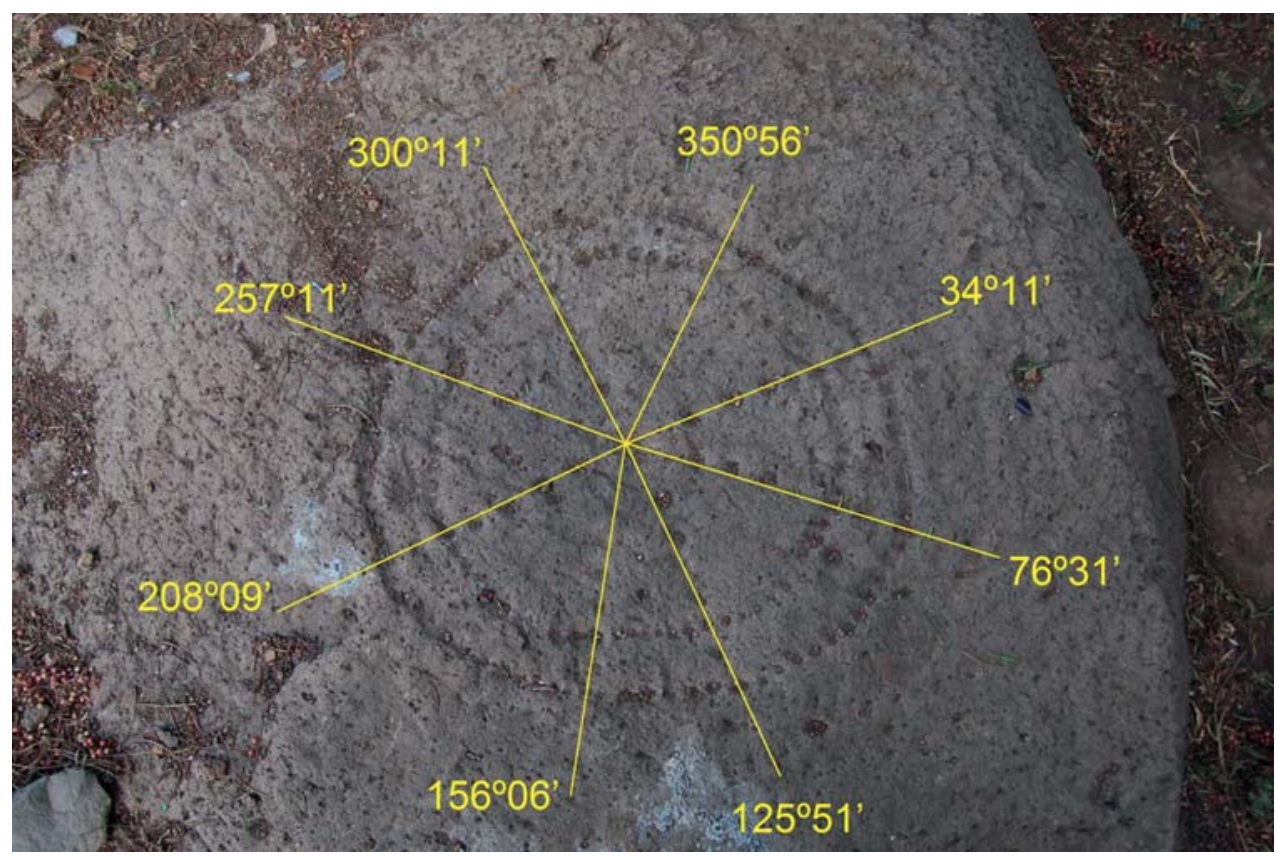

Figura 3b. Los acimuts de los ejes del marcador COC1. 
nes pequeñas del marcador implican que pudo haber sido visto por un reducido número de personas. El lugar donde se sitúa el marcador ofrece una amplia vista hacia el este y sur, en cambio las vistas hacia el norte y oeste quedan obstruidas por la cima de El Calvario. Se aprecia la vista a larga distancia en el perímetro acimutal de entre $15^{\circ}$ y $230^{\circ}$ lo que permite conjeturar que las referencias calendáricas y/o astronómicas del marcador tienen que ver con esta parte.

La figura tallada en roca constituye una imagen típica de la cruz punteada, por lo tanto siguiendo los argumentos de los investigadores citados arriba, se puede concluir que fue hecha durante el Clásico. Aunque en la época teotihuacana en la región de Chalco-Amecameca se notó el nivel bajo de la densidad poblacional (Parsons et al. 1982: 335), en el pie de monte bajo, al noroeste y al oeste del Cerro de Cocotitlán, se localizaron dos pequeñas aldeas (denominadas como una pequeña aldea dispersa (Ch-Cl-14) y una pequeña aldea nuclear (Ch-CL-15), respectivamente, (Parsons et al. 1982: 246). Es probable que ambas aldeas funcionaran como un centro administrativo local (Parsons et al. 1982: 332). Por otro lado, el área de Xico tuvo una ocupación teotihuacana sustancial, posiblemente relacionada con la agricultura de chinampas (Noyola 1993: 47-48). Aunque el cauce del río Tlalmanalco se encuentra relativamente cerca y puede usarse para las técnicas de irrigación, el terreno alrededor de Cocotitlán es mucho más apto para la agricultura de temporal. Sea como fuera, la presencia de ambas aldeas ( ¿de un centro administrativo de menor rango?) indica que la distancia entre el grabado y el asentamiento permanente más cercano se define por la caminata de unos 15-20 minutos.

Una gran cavidad en roca, de $4.5 \mathrm{~cm}$ de diámetro constituye la parte central de la figura. De ella salen en 8 direcciones diferentes los ejes acomodados en una secuencia de $4+(1)+1+(1)$ puntos. Su diseño es regular y tiende de ser uniforme, con la excepción del eje sur-sur-este) aunque las direcciones de los brazos no se encuentran dispuestas simétricamente (véanse Figuras $3 a$ y $3 b$ ).

El círculo interior cuenta con 61 orificios, dispuestos en 8 cuadrantes delimitados por los brazos descritos arriba. El círculo intermedio tiene 79 hoyos colocados en 7 cuadrantes. El círculo exterior contiene alrededor de 101 orificios. Este último círculo está parcialmente destruido en la parte sur y el número de orificios es solo aproximado.

$\mathrm{Al}$ admitir que los motivos punteados funcionan como contadores calendáricos, es decir, poseen algún valor numérico, se puede proponer que cada orificio representa una unidad, quizá de un día (en el sentido del periodo de 24 horas). Entonces los puntos colocados en los círculos representarían los cómputos de días, divididos en unidades menores delimitadas por los ejes. Los datos sobre los agujeros colocados en los círculos están reunidos en la Tabla 1.

La segunda premisa consiste en utilizar la línea de horizonte para observar las posiciones del sol saliente o poniente en ciertas fechas. Esta hipótesis propone que los ejes de los marcadores indican los puntos de salida o puesta de los cuerpos 
celestes seleccionados para determinar eventos calendáricos. Ello implica que para seleccionar los paneles rocosos adecuados para plasmar los marcadores, se tomaron en cuenta los puntos de salida o puesta en fechas determinadas. Aparte de esta hipótesis astronómica, se puede proponer la hipótesis topográfica, complementaria a la primera. No solo se trataría de fijar los puntos en el horizonte marcando las salidas o puestas del sol en fechas deseadas sino también de relacionar los marcadores con las determinadas formas de paisaje. En el horizonte oriental se aprecia la silueta de Iztaccíhuatl y sus elevadas cumbres cubiertas de nieve constituyen la forma del relieve más significativa. Su enorme silueta y contornos compuestos por los picachos de diferentes tamaños presentan una línea ondulante separando la Cuenca de México del Valle de Puebla. Los picachos reciben nombres de las partes del cuerpo humano que representan, formando la Cabeza, el Pecho, la Panza, las Rodillas y los Pies (véase Figura 4a). En la dirección sur-este se levanta el cono volcánico de Popocatépetl. Entre ambos volcanes se encuentran las cumbres menores de Cerro La Joya, Altzomoni, Venacho, Yoloxóchitl y Tlamacas. Al norte de la Cabeza se aprecian las cumbres del C. Tehuicocone, Teyotl, Papayo, Telapon y Tláloc. La forma del relieve presenta múltiples rasgos que con facilidad pueden usarse como marcadores de las salidas del sol en diferentes épocas del año. La gran parte de los cerros en la franja oriental se encuentra dentro del ángulo de desplazamiento anual del sol por el horizonte, lo que da soporte para la segunda premisa. Destaca el hecho de que la cadena de las cimas que conforman la silueta de Iztaccíhuatl permite fijar las salidas del sol solo durante la mitad del año (entre el equinoccio de otoño y el equinoccio de primavera, véase Figura 4b). Cabe subrayar que el este argumento puede ser importante porque los edificios mesoamericanos están desviados, en su mayoría, en la dirección sureste - noroeste (Šprajc 2001), o sea, en la dirección en donde se halla la silueta de Iztaccíhuatl en el caso de Cocotitlán. También se nota que durante el mes de febrero (y octubre/noviembre) se observan las salidas del sol detrás de los diferentes picachos que constituyen la silueta de Iztaccíhuatl. Las salidas del Sol en las fechas entre 16 y 19 de febrero (y 22-25 de octubre) ocurren cuando este astro se encuentra detrás del Pecho, mientras que las salidas del Sol en las fechas entre 8 y 12 de febrero ( 29 de octubre -2 de noviembre) suceden cuando se halla detrás la Panza (ver Fig. 4c). Cuando la imagen del Sol saliente abandona la Panza y sube a los largo del horizonte para aparecer detrás de El Pecho, es cuando se llega a dividir el año solar en dos partes: el periodo entre 12 de febrero y 29 de octubre tiene 260 días, y el periodo entre 30 de octubre y 11 de febrero contiene los restantes 105 días. Estas mismas orientaciones se encuentran definidas por la traza urbana en Teotihuacán (Iwaniszewski 1991; Šprajc 2000). Es de suponer que los antiguos habitantes de Cocotitlán fijaban las fechas más importantes del ciclo anual del sol mediante un sistema de puntos de referencia sobre el horizonte y la elección del lugar para colocar el marcador COC1 dependió de la observación de la cadena montañosa de Iztaccíhuatl con relación a los eventos astronómicos y climáticos. Al dirigir la mirada hacia la cadena de cimas que conforman la silueta Iztaccíhuatl y en 


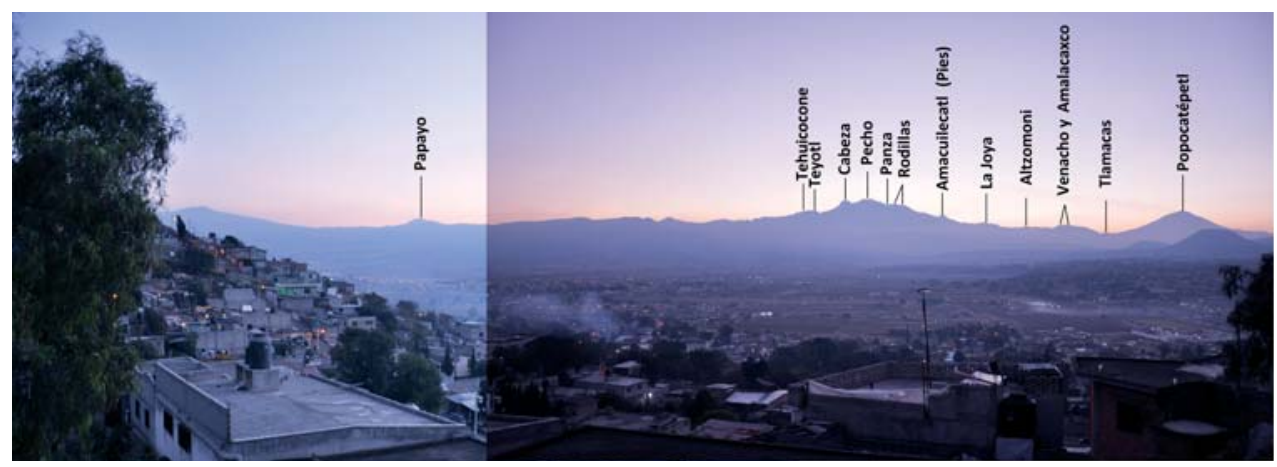

Figura 4a.Marcador COC1; vista hacia el este y sureste.

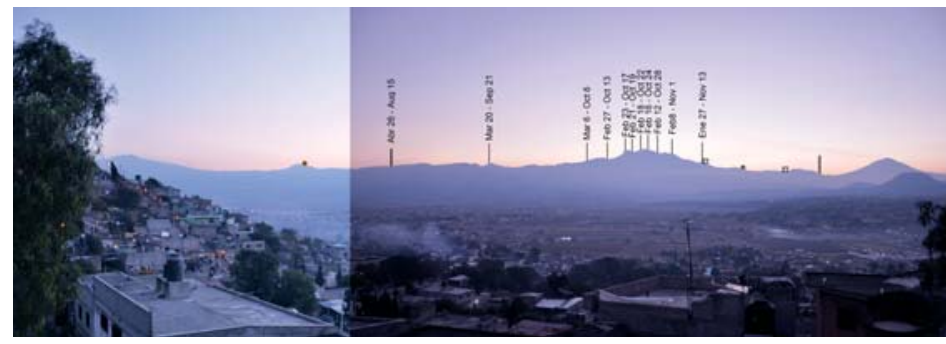

Calendario de horizonte

Cocotitlán

Marcador COC 1

Figura 4b. Marcador COC1; calendario de horizonte.
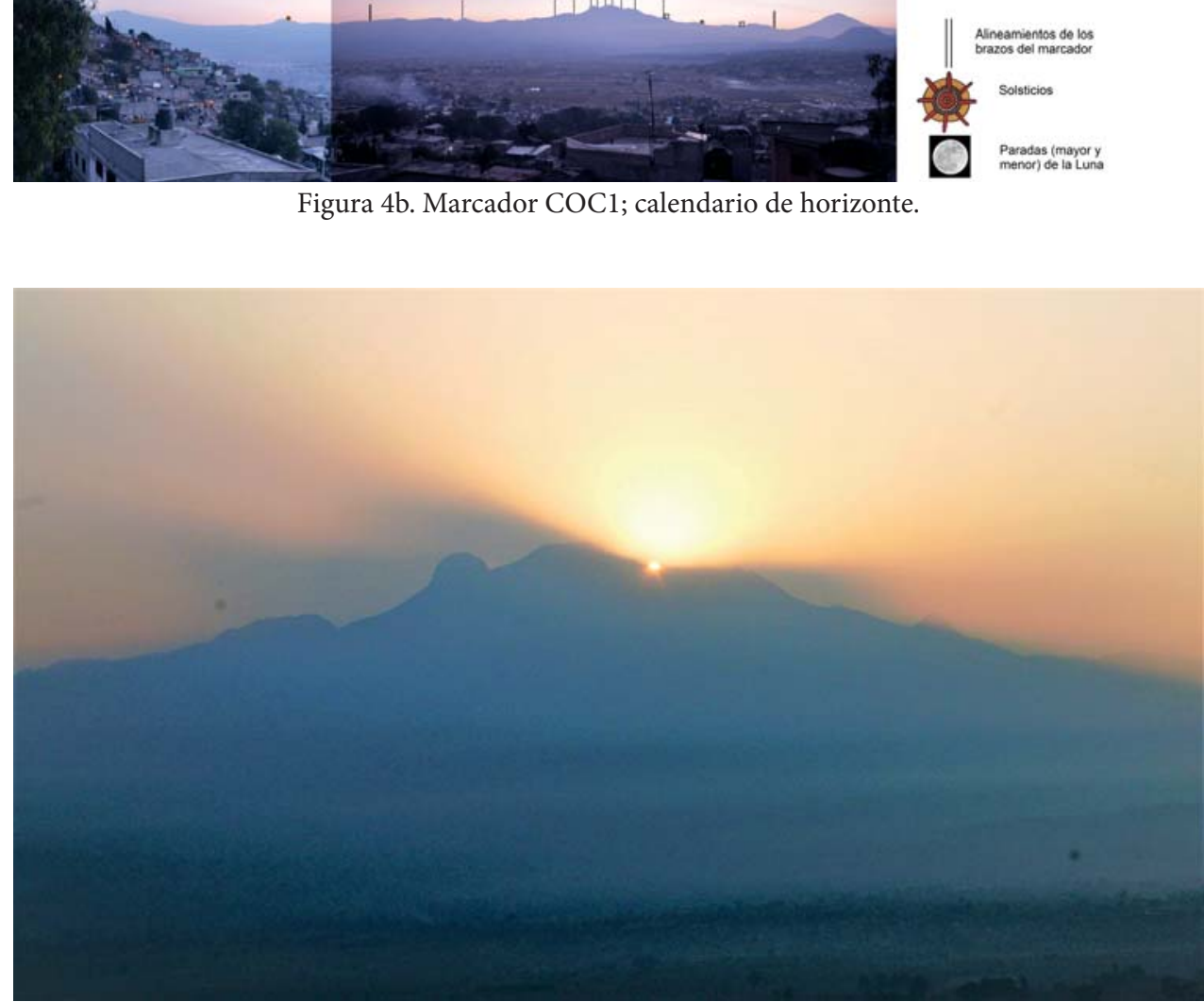

Figura 4c. Salida del sol observada el 12 de febrero de 2009 sobre el Pecho de la Iztaccíhuatl. 
donde se observa el sol saliente durante el mes de febrero, los hacedores del marcador pudieron construir las referencias a la orientación de la traza urbana en Teotihuacán. Naturalmente, siguiendo el ejemplo teotihuacano se podría concluir que la observación de las salidas consecutivas del sol sobre el macizo montañoso de Iztaccíhuatl (febrero y octubre) pudo determinar la duración del ciclo agrícola.

$\mathrm{Al}$ tomar en cuenta los alineamientos astronómicos de la figura, se nota que solo un brazo se encuentra dentro del ángulo de desplazamiento solar en el horizonte oriental. Su dirección marca las salidas del sol en las fechas 26/27 de abril y 15 de agosto $( \pm 1 \text { día })^{1}$ que dividen el año solar en los intervalos de 110 y 255 días. Justamente, cuando al salir el disco solar se halla detrás del Cerro Papayo, marca las fechas 29 de abril y 13 de agosto, que denotan ya la clásica división del año solar en los intervalos de 260 y 105 días ( \pm 1 día). El intervalo de 260/105 días aparece inscrito en la traza urbana teotihuacana y la dirección del brazo podría, en forma aproximada, marcar lo mismo. Sea lo que fuere, cabe mencionar que durante los primeros días de mayo (y de agosto) el sol está saliendo al norte del Cerro Papayo, detrás del paso del Río Frío que separa la Serranía del Cerro Tláloc (Tlalocán) del resto de la Sierra Nevada. Metafóricamente dicho, el sol sale sobre el Tlalocán durante la primera mitad de mayo ( $\mathrm{y}$ a finales de julio y principios de agosto) lo que puede relacionarse con el ciclo del crecimiento del maíz. Además el paso del Río Frío marca una hendidura visible en la línea del horizonte, si el sol sale allá, coincide con el tiempo cuando brotan las plantas del maíz y cuando se acerca la temporada de la lluvia. En otras palabras, la salida del sol detrás del Tlalocán denota el inicio de las lluvias.

En el Cerro Cocotitlán se localizan otros marcadores más. Meléndez García (2008: 100) reporta la presencia del marcador a los pies de la falda sureste del cerro, otro marcador se encuentra en la Casa de Cultura, cerca de El Calvario (véase Figura 4) y Galicia Cordova (1997: 68) publica la foto de un marcador más. Además, las entrevistas con los habitantes de Cocotitlán indican que existen todavía otros marcadores en la región.

Durante la época mexica el Cerro de Cocotitlán se convirtió en uno de los santuarios en los que se sacrificaban niños a los dioses del agua durante las ceremonias celebradas en el mes de Atlcahualo (correspondiente a febrero). Los motivos rupestres localizados en el promontorio a corta distancia del marcador COC 1 representan la cara de Tláloc, señalando que esta área perteneció al espacio ritual. La presencia de la silueta de Iztaccíhuatl que parece dominar la vista hacia el oriente, puede hacer referencia a su nombre prehispánico, el Tonacatépetl ("cerro del nuestro mantenimiento"), siendo el maíz y las demás plantas comestibles guardados en el interior de esta montaña (Serna 1953: 68). Hay que recalcar que las ceremonias de sacrificio de niños tuvieron el objetivo de propiciar la riqueza y abundancia tanto de las precipitaciones pluviales como de los alimentos. Toda esa evidencia arqueológica e histórica determina el contexto ritual del posible funcionamiento de los marcadores. 


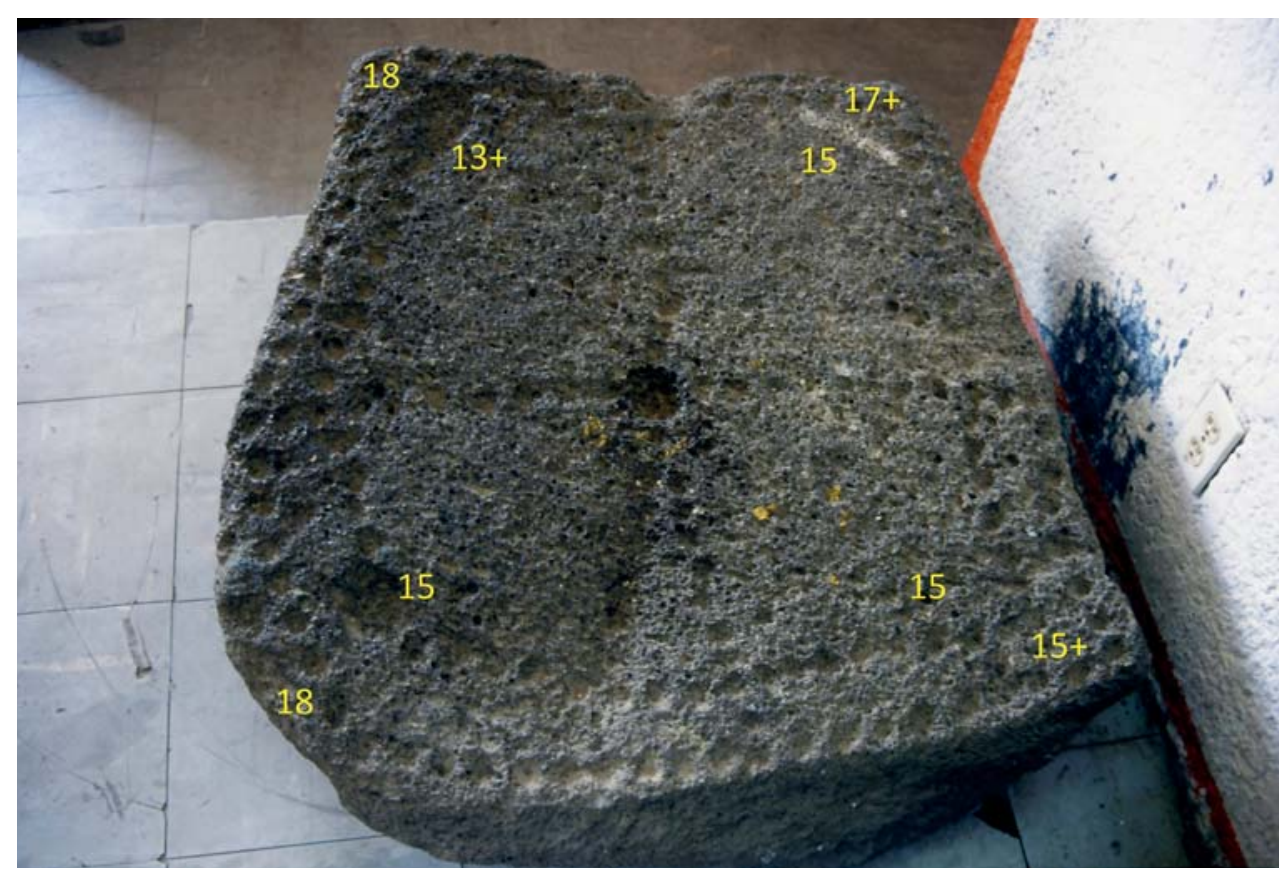

Figura 5. Marcador COC2.

Aunque se desconoce la naturaleza de las prácticas rituales en la cima del Cerro de Cocotitlán en la época teotihuacana, la presencia de los marcadores grabados en rocas indica que los antiguos habitantes seguían una tradición teotihuacana orientada a la observación y al estudio formal de los movimientos del Sol a lo largo de horizonte.

\section{$\operatorname{COC} 2$}

En la actualidad este marcador se encuentra en la Casa de Cultura, cerca de El Calvario (véase Fig. 5), fuera de su contexto arqueológico. Representa una figura regular compuesta por dos brazos y dos círculos concéntricos que tienen formas irregulares. Los orificios plasmados en el círculo interior suman a 58+, sin embargo, la parte dañada puede representar unas 2-3 cavidades más. Los agujeros del círculo exterior suman a $68+$.

\section{$\operatorname{COC} 3$}

Este marcador se sitúa en el afloramiento rocoso, en distancia de 2 kilómetros al sureste $\left(\mathrm{Az}=115^{\circ} 33^{\prime}\right)$ del marcador COC 1 , en el borde rocoso que enmarca el piedemonte volcánico, a la altura de $2285 \mathrm{msnm}$. Esta orientación casi coincide con la dirección solsticial. La cruz punteada se halla en la grada más baja que se sitúa en los lomeríos que descienden del sur y sureste y que se elevan tan solo 6-8 metros 


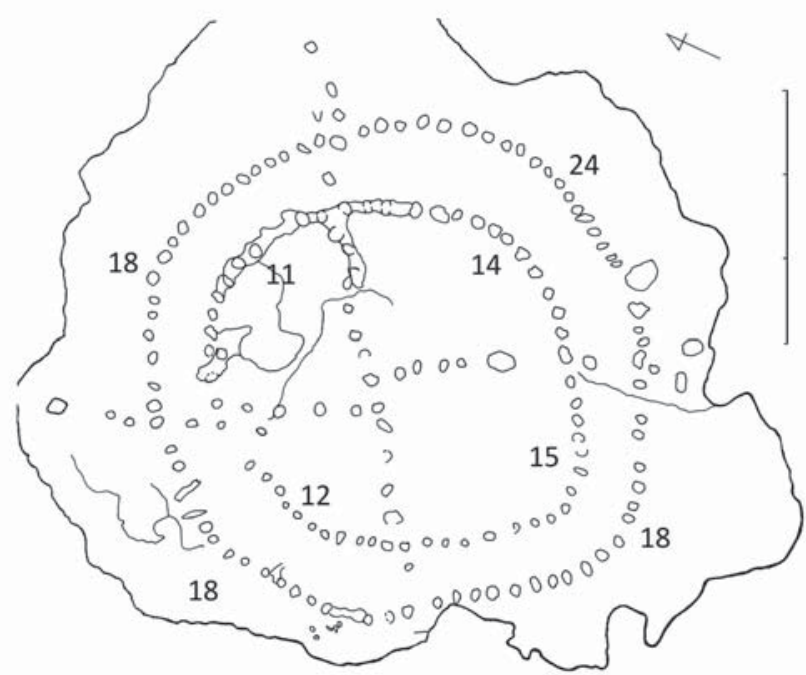

\section{$\operatorname{COC} 3$}

Levantamiento y Dibujo:

S. Iwaniszewski

Fecha: 7.12.2013

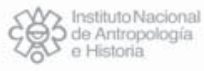

Figura 6a. El diseño del marcador COC3.

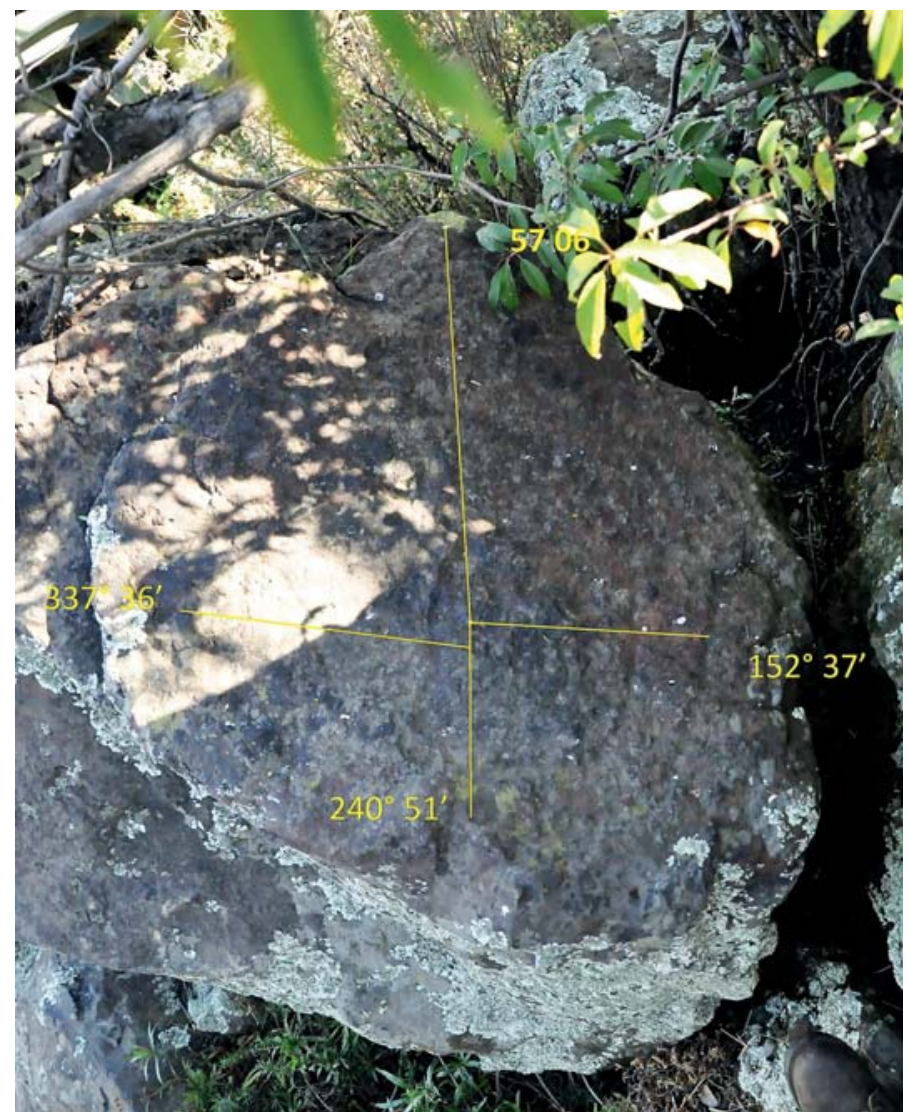

Figura 6b. Los acimuts de los ejes del marcador COC3. 


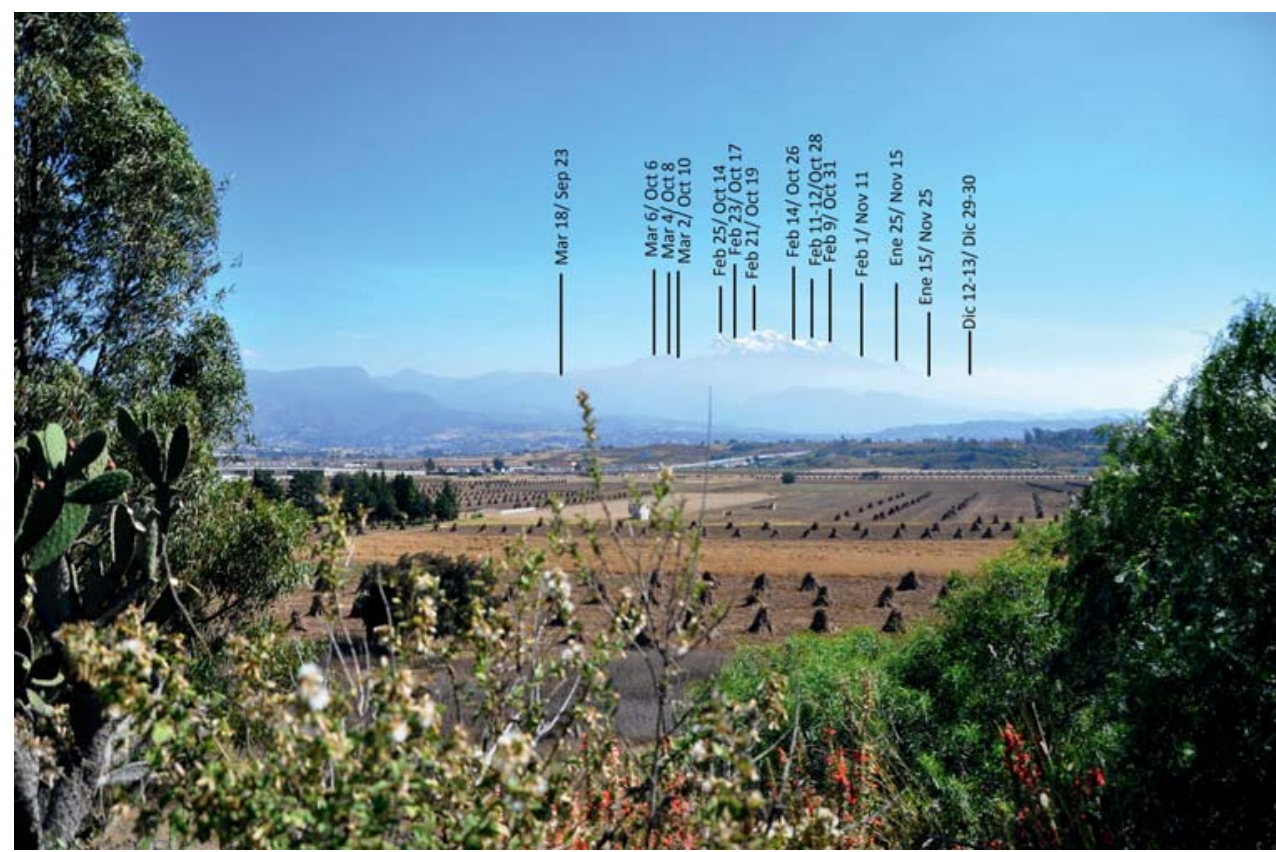

Figura 6c. Marcador COC3; calendario de horizonte.

arriba de la planicie flanqueada en el norte por el lecho del Río Tlalmanalco. En la actualidad este afloramiento rocoso está rodeado por los campos de cultivo. El marcador está hecho en la superficie rocosa, inclinada entre $20^{\circ}$ y $30^{\circ}$ hacia el sureste (la dirección de Iztaccíhuatl). El lugar ofrece una excelente vista hacia el norte y oriente, en el perímetro de $250^{\circ}$ y $125^{\circ}$, mientras que las vistas hacia el poniente y hacia el sur quedan bloqueados los lomeríos que descienden del piedemonte volcánico (véase Fig. 6c). Sin embargo, basta subir a la cima del afloramiento, unos 2 metros arriba, para encontrar la vista panorámica de casi $360^{\circ}$. El marcador se encuentra en la distancia de 15 minutos a pie del asentamiento teotihuacano más cercano. Finalmente cabe hacer notar que ambos marcadores, el COC 1 y e COC 3 son inter-visibles.

La figura mide $77 \mathrm{~cm}$ (eje NO-SE) por $69 \mathrm{~cm}$ (eje NE-SO), el diámetro del círculo exterior mide entre 58 y $64 \mathrm{~cm}$, el diámetro del círculo inferior tiene entre 41 y $48 \mathrm{~cm}$. Es importante señalar que el brazo NO-SE no es continuo (véanse Fig. 6a y 6b). Los brazos no apuntan hacia las salidas o puestas del Sol. El círculo interior tiene $52+$ orificios preservados, el círculo exterior contiene 78 orificios, los brazos se tienen 20 orificios preservados (ver Fig. $6 \mathrm{a}$ y $6 \mathrm{~b}$ )

En el mismo lugar se hallaron las representaciones de Tláloc, que juzgando por su estilo, se pueden atribuir al Postclásico. Este hecho indica que el mismo lugar fue re-utilizado y apropiado para el ritual agrícola. 


\section{La descripción estructural de los marcadores en Cocotitlán}

Los datos que se exhiben en la Tabla 1 determinan los números de los orificios. Las cantidades fueron determinados a partir de las calcas y fotografías in situ. Las superficies rocosas en donde se plasmaron los marcadores sufrieron varios daños lo que afecta las interpretaciones. Los números que aparecen en la última columna describen las cantidades de los orificios reconstruidos (ver Tabla 1).

\section{$\operatorname{COC} 1$}

La parte central de la figura la constituye un gran orificio central, de $4.5 \mathrm{~cm}$ de diámetro. Del orificio parten en 8 direcciones diferentes los brazos (ejes) que constan de 4 hoyos cada uno. Su diseño es regular, aunque las direcciones de los brazos no se encuentran dispuestas simétricamente (véase Fig. 2a y 2b). Uno de los brazos parece chocar con el círculo interior y no tener ninguna continuidad. Creo que eso se debe a la destrucción porque tomando en cuenta la disposición regular de los orificios en los brazos todos los brazos chocan con el círculo intermedio. Los ejes manifiestan el patrón regular de 4 - (1) - 1- (1) puntos (entre paréntesis se señalan las intersecciones), solo al brazo sur-sur-este le falta el orificio entre el círculo interior y el intermedio. De ser así, tendríamos en total $8 \times 7=56$ puntos más el orificio central, en total 57 puntos (ver Fig. 3a). Es probable que los brazos no se prolonguen hacia el círculo exterior.

El círculo interior contiene en total 61 puntos repartidos en 8 secciones separados por los ejes de la figura.

Ahora bien, al reconstruir el brazo sur-sur-este, cabe la posibilidad de extenderlo para que choque con el círculo intermedio. Para mantener la disposición de los orificios de este brazo, el patrón de 4 - (1) - 1 - (1) puntos, es necesario restar un orificio del círculo intermedio. De tal manera el conjunto de 19 orificios se divide en grupos menores, de 6 y 12 puntos, tal como se aprecia en las secciones a la derecha. Por tanto, el círculo intermedio ahora contiene 78 hoyos. Ambos círculos contienen 139 orificios. El círculo exterior contiene alrededor de 101 orificios. Este círculo está parcialmente destruido en la parte sur y el número de orificios es solo aproximado (véanse Fig. 3a y Tabla 1).

\section{$\operatorname{COC} 2$}

Este marcador consta de dos círculos centrados a una cruz cartesiana. Los brazos parecen tener el siguiente patrón; 8 - (1) - 1 - (1), en total 44 (o 45 puntos incluyendo el agujero central).

La forma de ambos círculos está distorsionada, creando líneas rectas en lugar de arcos. Parece que el diseño original fue ajustado a las dimensiones del bloque de roca en donde se plasmó el marcador. Las reconstrucciones de las partes dañadas permiten establecer, de manera hipotética, las cantidades de orificios faltantes (Fig. 5). El círculo interior contiene $15,15,13+15,=58+$ orificios. Es posible que tenga 15, 15, 
15, 15 = 60 hoyos. El círculo exterior cuenta con $15+18,18,17+=68+$ orificios, pero podría tratarse de $18,18,18,18=72$ orificios (ver Tabla 1 ). Ambos círculos presentan $127+$ orificios, pero la reconstrucción indica que podrían contar con 132 puntos. En total la figura contiene alrededor de 177 puntos.

\section{$\operatorname{COC} 3$}

El marcador representa dos círculos centrados en el cuasi-eje cartesiano: uno de los brazos no parte del centro común. Los brazos exhiben el patrón diferenciado de orificios, de 5 - (1) - 1 - (1), es decir, $8 \times 4=32$ (¿?) puntos (véase Fig. 6a). Tres de los ejes parecen extenderse al exterior del círculo exterior, por lo tanto, el número de los orificios puede elevarse. El círculo interior contiene 15, 12+, 11+, 14+ = 52+ puntos, pero algunos de los orificios fueron destruidos. Ya que el cuadrante sur-oeste parece ser el menos destruido de todos, se podría asumir que los demás cuadrantes originalmente tuvieron un número semejante de los hoyos. De ser así, el círculo interior contendría 60 orificios. El menos destruido el círculo exterior es muy regular y consta de 18, 18, 18, $24=78$ orificios (véanse Fig. 6a y 6b). En la actualidad ambos círculos tienen $130+$ orificios, es posible que contuviesen 138 orificios.

\section{Discusión}

En suma, los tres marcadores analizados presentan las cantidades semejantes de los orificios: 61, 59+, 52+ (círculo interior) y 79, 68+, 78 (círculo exterior). En mi opinión las figuras demuestran cierta similitud en la estructura de los números de orificios y pueden haber tenido una función similar. Las reconstrucciones recalcan aún más estas similitudes: 61, 60, 60 (círculo interior) y 78, 72, y 78 (círculo exterior). Ya que estos números de orificios grabados en piedra son también parecidos a los números de días evidenciados en los almanaques calendáricos en los códices del periodo Postclásico, se probable que las cuentas de los orificios de los marcadores representan los cómputos calendáricos.

Los marcadores de la región de Cocotitlán exhiben el patrón que difiere un poco de las cruces punteadas teotihuacanas. Los ejes de los marcadores teotihuacanos demuestran la tendencia del siguiente orden: 10-(1)-4-(1)-4, en total 20 agujeros, mientras que los marcadores COC 1, COC 2 y COC 3 exhiben el patrón de 5-(1)1-(1), en total 8 cavidades. Los agujeros en el círculo interior tienden a marcar los números entre 11 y 16 (en el caso de COC 1 se cuentan los sectores vecinos) y en el círculo exterior oscilan entre $15+$ y 24 . Se puede especular que la suma de los agujeros en el círculo interior se sitúa cerca de 60 puntos, mientras que en el círculo exterior tiende a tener alrededor de 78 puntos.

Los calendarios del horizonte y los marcadores de Cocotitlán

En el oriente se aprecia la silueta de Iztaccíhuatl y sus elevadas cumbres cubiertas de nieve constituyen la forma del paisaje que parece ser la más significa- 
tiva (véase Fig. 4a). Su enorme silueta y contornos compuestos por los picachos de diferentes tamaños presentan una línea ondulante separando la Cuenca de México del Valle de Puebla. Los picachos reciben nombres de las partes del cuerpo humano que representan, formando la Cabeza, el Pecho, la Panza, las Rodillas y los Pies. En la dirección sur-este se levanta el cono volcánico de Popocatépetl. Entre ambos volcanes se encuentran las cumbres menores de Cerro La Joya, Altzomoni, Venacho, Yoloxóchitl y Tlamacas. Al norte de la Cabeza se aprecian las cumbres del C. Tehuicocone, Teyotl, Papayo, Telapon y Tláloc. La forma del relieve presenta múltiples rasgos que con facilidad pueden usarse como marcadores de las salidas del sol en diferentes épocas del año (ver Figuras 4 b y $6 c$ ). Las salidas del Sol detrás de la Panza (vistas desde el COC 1) o detrás de la Rodillas (vistas desde el COC 3) suceden en las fechas 11-12 de febrero y 28-29 de octubre, dividiendo el año solar en 2 partes: de 260 días (del 11 de febrero al 29 de octubre) y 105 días (del 30 de octubre al 11 de febrero). Estas mismas orientaciones se encuentran definidas por la traza urbana en Teotihuacán. Las salidas del Sol sobre las cumbres más elevadas de Iztaccíhuatl informaban a los habitantes de las aldeas del periodo Clásico sobre su afiliación teotihuacana.

Tomando en cuenta el cronograma actual de las actividades campesinas, se puede asociar el inicio de la cosecha (lo que hoy ocurre después del Día de los Muertos) con las posiciones del orto solar a la derecha del todo el macizo de Iztaccíhuatl (al sur de las Rodillas). Cuando el Sol se aleja paulatinamente de Iztaccíhuatl y se mueve hacia el Popocatépetl ocurre la recolección del maíz. Cuando el Sol vuelve a salir sobre el Iztaccíhuatl, indica que ya es el tiempo de la selección de milpas y de su preparación para la siembra. Quizá por esas razones, el Cerro Cocotitlán fue seleccionado por los mexicas para celebrar los sacrificios de niños en el mes de Atlcahualo (12 de febrero - 4 de marzo). Hay que recalcar que en el siglo XVI la montaña de Iztaccíhuatl fue conocida como Tonacatépetl ("cerro del nuestro mantenimiento"), siendo el maíz y las demás plantas comestibles guardados en el interior de esta montaña (Serna 1953: 68) y que las ceremonias de sacrificio de niños tuvieron el objetivo de propiciar la riqueza y abundancia tanto de las precipitaciones pluviales como de los alimentos. Probablemente esta percepción de los fenómenos astronómicos, calendáricos y climáticos formó parte de la experiencia religiosa articulando una vez más la relación entre la vida del hombre y los ciclos temporales presididos o controlados por númenes o entidades espirituales. Los vínculos espaciales entre las formas del paisaje eran inteligibles cuando las relaciones visuales entre los cerros fueron reveladas por el movimiento solar. La ubicación del marcador que sirve para realizar cómputos calendárico-astronómicos cobra importancia cuando termina un ciclo de 260 días y arranca el siguiente, o cuando concluye un ciclo temporal y se inicia otro. Cabe recalcar que la ubicación del marcador permite mirar directamente en la dirección de Iztaccíhuatl, mientras que la vista hacia la dirección en donde sale el Sol durante el solsticio de verano está un poco sesgada (véase Figura $4 \mathrm{~b}$ ). 
¿Existe alguna relación entre los orificios del marcador y las fechas de la salida del Sol en el horizonte oriental? El eje del marcador COC 1 apunta al orto solar en 26 de abril y 15 de agosto indicando el periodo 20 días antes y después del paso cenital (16 de mayo y 26 de julio). Se observa que el intervalo de 78 días, cercano a la suma de los agujeros del círculo intermedio, separa las fechas de los ortos solares sobre la Rodilla en el Iztaccíhuatl ( 8 de febrero y 1 de noviembre). El lapso de 61 días (el número de los orificios del círculo interior) denota el intervalo que se puede encontrar entre la salida del Sol sobre el eje del marcador (26 de abril/15 de agosto) y su salida debajo de la Cabeza (25 de febrero/15 de octubre).

Desafortunadamente, los ejes del marcador COC 3 no marcan las fechas solares. El intervalo de 60 días puede denotar el lapso entre la fecha de la salida del Sol sobre el Pecho, la cumbre más alta de Iztaccíhuatl (19 de febrero/21 de octubre) y el solsticio de diciembre ( 22 de diciembre). Cualquier otra interpretación sería en este momento una mera conjetura. No obstante, la correspondencia entre las fechas y las siluetas de las montañas que constituyen el calendario de horizonte es muy semejante a la del marcador COC 1.

A pesar de estas limitaciones, se puede proponer el punto de vista diferente. Los marcadores COC 1 y COC 3 contienen las cantidades de los orificios dispuestos en los círculos muy semejantes, 61 o 60 para el círculo inferior y 78 para el círculo superior. El número 60 es divisible entre 20 ( 3 x 20), mientras el número 78 es divisible entre 13 (6 x13) y ambos números aparecen en los cómputos calendáricos en los códices (ver Iwaniszewski 2005). Tomando como explicación las propuestas de Jesús Galindo (2004) y Hans Martz de la Vega y Miguel Pérez Negrete (2014) sobre un patrón global mesoamericano organizado a partir de la división del año días en trecenas y veintenas partiendo del día del solsticio de invierno, se podría aplicarlas al presente análisis. Aplicando esta propuesta al estudio de los marcadores de la región de Cocotitlán, se puede plantear que el propósito de las figuras COC 1 y COC 3 consistió en marcar los intervalos de 60 y 78 días partiendo del día del solsticio de invierno.

Para ambos intervalos de días el pivote solsticial cae en los días 21-22 de diciembre. Por lo tanto, la diferencia de 60 días con respecto al solsticio de invierno será el 19-20 de febrero/22-23 de octubre. Estas fechas coinciden con las posiciones del Sol cerca del Pecho (véanse Fig. 4b y Fig. 6c). A su vez, la diferencia de 78 días con respecto al solsticio de invierno denota las fechas 9-10 de marzo/4-5 de octubre. Se observa que en estos días el Sol se encuentra a la izquierda de la cresta conformada por los cerros Tehuicocone y Teyotl. Es decir, el Sol se encuentra justamente antes de subir al macizo de Iztaccíhuatl (o después de bajar de él). Al mismo momento se observa que estas fechas coinciden con eventos agrícolas importantes. Las fechas febrero-marzo se asocian con los inicios de la siembra, las fechas de octubre señalan que el crecimiento del maíz se acerca a su fin (véase Fig. 2).

Por último, la presencia del intervalo de 78 días parece indicar que los hacedores y usuarios de los marcadores estaban familiarizados con la división del año solar 
en 28 trecenas (= 364 días, el llamado "año computacional”). Según las propuestas de Galindo, Martz de la Vega y Pérez Negrete, los intervalos de días como múltiplos de trece fueron ideados para orientar la arquitectura prehispánica en Mesoamérica. En este contexto es interesante señalar que los diseñadores de los marcadores utilizaron un esquema semejante para marcar las fechas propicias para ciertas faenas agrícolas.

\section{Conclusiones}

En la Cuenca de México varos marcadores fueron colocados sobre las superficies rocosas en lugares previamente seleccionados, invisibles desde lejos, con vistas limitadas hacia el valle, lo que me impulso a proponer de qué se trataba de los sitios apropiados para representar el saber calendárico, astronómico y ritual especializado y local. Además, su cercanía a los campos de cultivo puede indicar que conformaban los sitios en donde se celebraban algunos rituales agrícolas con un fuerte contenido calendárico-astronómico (Iwaniszewski 2006).

Para las sociedades agrícolas asentadas en las aldeas en la parte sur de la Cuenca de México, los rasgos físicos sobresalientes del medio ambiente circundante pudieron fácilmente convertirse en los vehículos de las ideas religiosas, cosmovisionales e ideológicas relacionadas con el cultivo del maíz. La línea ondulante conformada por la silueta de los cerros pertenecientes a la Sierra Nevada ofreció la posibilidad de observar las correspondencias entre las fechas de las posiciones del Sol sobre el horizonte y los ciclos climáticos y agrícolas. Al morar o habitar esta parte de la Cuenca, ellos se habituaron a percibir las siluetas de la cadena montañosa, incluyendo la cadena de las cumbres que hoy constituyen Iztaccíhuatl. La permanencia en la región les permitió familiarizarse con la imagen del Sol saliente y asociar sus posiciones particulares con los cambios estacionarios y las tareas agrícolas importantes. La fijación de los alineamientos específicos se hizo mediante la traza de los marcadores. Las relaciones con el paisaje terrestre y celeste fueron codificadas por el calendario y en especial, por los intervalos numéricos a través de los cuales estas relaciones encontraron su expresión. En cierto sentido, los sitios discutidos en este artículo se sitúan en lugares privilegiados para crear o percibir este tipo de relaciones.

Es de esperar que los especialistas en cómputos calendáricos responsables por el diseño y la elección de los lugares en donde se plasmaron los marcadores, se dieran cuenta de las asociaciones que se daban entre las localidades cerca de Cocotitlán y el desplazamiento de los ortos solares en febrero y octubre a lo largo de la enorme silueta de Iztaccíhuatl. La falda occidental de Iztaccíhuatl está cubierta por los densos bosques y profundos cañones que transportan aguas de deshielos, ambos recursos importantes para la vida económica de las sociedades agrarias acomodadas en el Valle Chalco-Amecameca. Los eventos solares parecen dar el sentido al transcurso del tiempo, ordenando las formas del paisaje en función de los ritmos ambientales y labores en la milpa. Dos veces al año el disco solar recorre un gran número de cerros 
que conforman Iztaccíhuatl incorporando el conteo de días a las actividades rituales uniendo el tiempo del cosmos con el tiempo del hombre. Desconocemos la naturaleza de las prácticas rituales en la cumbre del Cerro de Cocotitlán en la época teotihuacana. No obstante, la presencia de los marcadores grabados en rocas indica que los antiguos habitantes seguían una tradición teotihuacana orientada a la observación $\mathrm{y}$ al estudio formal de los movimientos del Sol a lo largo de horizonte. Las investigaciones futuras deben tomar en cuenta estas implicaciones para la reconstrucción del paisaje ritual en Cocotitlán.

\section{Agradecimientos}

Mis agradecimientos para el Dr. Adán Meléndez García (INAH) por señalarme la colocación del marcador COC3. Agradezco también al comentario del revisor anónimo. El trabajo fue realizado como parte del proyecto "La montaña en el paisaje ritual” registrado en el Instituto Nacional de Antropología e Historia.

\section{Notas}

1 Los orificios colocados en las intersecciones se asignan como pertenecientes a los ejes (véanse Aveni 1989, Iwaniszewski 1991).

2 Ya que los orificios no se sitúan en una línea recta, por lo general se mide la línea que une el centro de la figura con el centro de uno de los orificios más alejados.

Aveni, Anthony F.

1989 Pecked Cross Petroglyphs at Xihuingo. Archaeoastronomy 14 (supplement to the Journal for the History of Astronomy, vol. 20): S73-S115.

1991 Mapping the Ritual Landscape: Debt Payment to Tlaloc During the Month of Atlcahualo. En To Change Place: Aztec Ceremonial Landscapes, editado por Davíd Carrasco, pp. 58-73. University Press of Colorado, Niwot.

Broda, Johanna

1991 The Sacred Landscape of Aztec Calendar Festivals: Myth, Nature, and Society. En To Change Place: Aztec Ceremonial Landscapes, editado por Davíd Carrasco, pp. 74-120. University Press of Colorado, Niwot.

Cabrera Castro, Rubén

2002 Teotihuacan Cultural Traditions Transmitted into the Postclassic According to Recent Excavations. En Mesoamerican Classic Heritage. From Teotihuacan to the Aztecs, compilado por Davíd Carrasco, Lindsay Jones y Scott Sessions, pp. 195-218. University Press of Colorado, Boulder. 
Díaz Cisneros, Heliodoro y Enrique Cruz López

1993 El cultivo del maíz en la economía familiar campesina en la región Chalco - Amecameca. En: Entre lagos y volcanes. Chalco Amecameca: pasado y presente, editado por Alejandro Tortolero, Vol. I, pp. 521-574. El Colegio Mexiquense, Zinacatepec.

Galicia Cordova, Arturo

1997 Cocotitlán. Monografía Municipal. Gobierno del Estado de México, Instituto Mexiquense de Cultura, Toluca.

Galindo Trejo, Jesús

2004 Ordenamiento calendárico de la arquitectura mesoamericana. La pintura mural prehispánica en México, vol. 20: 16-20.

Iwaniszewski, Stanisław

1991 La arqueología y la astronomía en Teotihuacán. En Arqueoastronomía $y$ etnoastronomía en Mesoamérica, editado por Johanna Broda, Stanislaw Iwaniszewski y Lucrecia Maupomé, pp. 269-290. Instituto de Investigaciones Históricas, Universidad Nacional Autónoma de México, México.

2005 Leer el tiempo. El Fenómeno de la sincronicidad en la práctica mántica teotihuacana. En Perspectivas de la Investigación Arqueológica, editado por Walburga Wiesheu y Patricia Fournier, pp. 93-108. Escuela Nacional de Antropología e Historia, Instituto Nacional de Antropología e Historia, México.

2006 Out of Teotihuacan: Cross-Circle Figures in the Valley of Mexico. En Viewing the Sky Through Past and Present Cultures. Selected Papers form the Oxford VII International Conference on Archaeoastronomy, editado por Todd W. Bostwick y Bryan Bates, pp. 213-224. Pueblo Grande Museum Anthropological Papers No.15. City of Phoenix and Recreation Department, Phoenix.

Martz de la Vega, Hans y Miguel Pérez Negrete

2014 Tehuacalco como lugar de memoria. Arqueoastronomía y paisaje. Región Centro de Guerrero. Cuicuilco 61: 303-331.

Meléndez García, Adán

2008 La permanencia en el paisaje del santuario prehispánico de Cocotitlan. En Festines y Ritualidades. Arqueología y Antropología de las Religiones, Volumen II, editado por Patricia Fournier y Walburga Wiesheu, pp. 93108. Escuela Nacional de Antropología e Historia, Instituto Nacional de Antropología e Historia - Promep, México, D.F.

Noyola, Jaime

1993 Xico: una aproximación al área chalca. En Entre lagos y volcanes. Chalco y Amecameca: pasado y presente, editado por Alejandro Tortolero, pp. 19-72. El Colegio Mexiquense, A.C. Zinacantepec, Edo de México. 
Parsons, Jeffrey R. Elizabeth Brumfiel, Mary H. Parsons y David J. Wilson

1982 Prehispanic Settlement Patterns in the Southern Valley of Mexico: The Chalco-Xochimilco Region. Memoirs of the Museum of Anthropology 14. University of Michigan, Ann Arbor.

Sahagún, Fr. Bernardino de

1979 Historia General de las cosas de Nueva España. [Sepan cuantos... núm. 300]. Editorial Porrúa, México

Serna, Jacinto

1953 Manual de ministros de indios para el conocimiento de sus idolatrías y extirpación de ellas. En Tratado de las idolatrías, supersticiones, dioses, rotos, hechicerías y otras costumbres gentilicias de las razas aborígenes de México, editado por Francisco del Paso y Troncoso, pp. 47-368. Ediciones Fuente Cultural, México.

Šprajc, Ivan

2000 Astronomical Alignments at Teotihuacan, Mexico. Latin American Antiquity 11(4): 403-415.

2001 Orientaciones astronómicas en la arquitectura prehispánica del centro de México. Colección Científica 427, Instituto Nacional de Antropología e Historia, México.

Tellos Charles, Norma Leticia

1993 Las expresiones rupestres de la Cuenca de México. Tesis de Licenciatura, Escuela Nacional de Antropología e Historia, México.

Vivó, Jorge A., José C. Gómez, Dolores Riquelme y Esperanza Yarza

1946 Climatología de México. Publicación 19. Instituto Panamericano de Geografía e Historia, México D.F.

Winning, Hasso von

1987 La iconografía de Teotihuacán: los dioses y los signos. Vol. 2. Instituto de Investigaciones Estéticas, Universidad Nacional Autónoma de México, México. 
